\title{
Budgethilfe und Steuerreform: Fallbeispiel Mosambik
}

Jean-Luc Bernasconi

\section{(2) OpenEdition}

\section{Journals}

Electronic version

URL: http://journals.openedition.org/sjep/409

DOI: $10.4000 /$ sjep.409

ISSN: 1663-9677

\section{Publisher}

Institut de hautes études internationales et du développement

\section{Printed version}

Date of publication: 1 novembre 2007

Number of pages: $225-226$

ISBN: 978-2-88247-069-0

ISSN: $1660-5926$

Electronic reference

Jean-Luc Bernasconi, «Budgethilfe und Steuerreform: Fallbeispiel Mosambik», Schweizerisches Jahrbuch für Entwicklungspolitik [Online], 26-2 | 2007, Online erschienen am: 03 Juni 2010, abgerufen am 08 September 2020. URL : http://journals.openedition.org/sjep/409 ; DOI : https://doi.org/ $10.4000 /$ sjep.409 


\title{
Budgethilfe und Steuerreform: Fallbeispiel Mosambik
}

\author{
Jean-Luc Bernasconi*
}

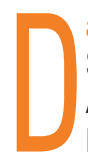
as SECO hat den Reformbemühungen der Steuerbehörden relativ rasch besondere Aufmerksamkeit zukommen lassen. Dadurch wird ein doppeltes Ziel angestrebt: Zum einen zur nachhaltigen Stärkung einer Finanzierungsquelle des Staatsbudgets beizutragen, das auf die Dauer einen „erfolgreichen" Ausstieg aus den Budgethilfeprogrammen ermöglichen sollte; zum anderen die Einführung einer wirklichen „Entwicklungsbesteuerung " $\mathrm{zu}$ unterstützen, das heisst eines Systems, bei dem Willkür und Korruption beseitigt sind und die Steuerbelastung kein Hindernis für Investitionen und Wachstum darstellt, wobei aber gleichzeitig Prinzipien der Chancengleichheit eingehalten werden. In Ländern, in denen ein grosser Teil der Wirtschaft vom informellen Sektor verwaltet wird, sind solche Ziele ambitiös und nur auf lange Sicht zu erreichen. Allgemein unterscheidet man zwischen der Reform der Steuerpolitik (z.B. Änderung der Steuersätze oder Übergang zur Mehrwertsteuer) und den administrativen Reformen mit dem Ziel, die Fähigkeit der Verwaltungsbehörden zur Umsetzung dieser Politik zu stärken, auch wenn politische und administrative Reformen letztendlich eng miteinander zusammenhängen. Die vom SECO unterstützten Programme fallen hauptsächlich unter die zweite Kategorie, wenngleich die Schweiz im Rahmen der Absprachemechanismen zur Budgethilfe ebenfalls am Dialog über Steuerpolitik beteiligt ist.

In Mosambik war die Steuerpolitik in den letzten Jahren - wie in vielen afrikanischen Ländern - eng mit der Handelspolitik verbunden. Die Massnahmen zur Handelsliberalisierung haben die Zolleinnahmen schrumpfen lassen, die bis vor kurzem die Mehrheit der Budgeteinnahmen ausmachten. Die interne Besteuerung (direkte Einkommenssteuern, indirekte Verbrauchssteuern oder -abgaben und "nichtsteuerliche" Einnahmen wie Bergbaulizenzen) muss somit die Verluste der externen Besteuerung (Zölle) ausgleichen, um das Niveau prioritärer öffentlicher Ausgaben aufrechtzuerhalten bzw. anzuheben. Zu diesem Zweck hat Mosambik Anfang des 21. Jahrhunderts ein umfangreiches Steuerreformprogramm in die Wege geleitet, indem es 1999 eine Mehrwertsteuer einführte, die mit weiteren klassischen Verwaltungsmassnahmen zur Effizienzsteigerung bei der Steuereinziehung einherging: Neuverteilung der Dienststellen entsprechend der Grösse der Steuerzahler, Verbesserung der Informationssysteme betreffend Steuerzahler, Reformen der Einkommensund Gewinnbesteuerung usw. Die Entwicklungsstrategie des Landes sieht eine Erhöhung der Budgeteinnahmen um 0,5 Prozent pro Jahr über den Zeitraum 2005-2009 vor. Zum anderen hat Mosambik - sozusagen nach angelsächsischem Muster - beschlossen, sämtliche Steuerdienststellen seit 2006 zu einer einzigen halbautonomen Institution, der zentralen Steuerbehörde (Autoridade Tributária Moçambicana, ATM), zusammenzufassen. Welche Ergebnisse wurden bislang erzielt? Der Steuersatz, der Ende der 90er-Jahre noch unter 10 Prozent des Bruttoinlandsprodukts (BIP) gelegen hatte, ist allmählich angestiegen und erreichte 2006 - trotz eines

* Leiter des Ressorts Makroökonomische Unterstützung, Bereich Wirtschaftliche Zusammenarbeit und Entwicklung, Staatssekretariat für Wirtschaft (SECO), Bern.

1 Der Begriff „Besteuerung“ wird hier im weiteren Sinne - d.h. als alle Arten von Budgeteinnahmen umfassend - angewandt: Steuern, Gebühren, Verbrauchssteuern sowie beispielsweise von Gebühren auf die Bewirtschaftung natürlicher Ressourcen abgeleitete Abgaben. 
hohen BIP-Niveaus - 14,4 Prozent des BIP. Somit ist zu begrüssen, dass die Steuerleistung selbst mit den erhöhten Budgethilfebeiträgen jedenfalls nicht zurückgegangen ist. Jedoch sind diese Steuersätze, auch im regionalen Vergleich, weiterhin niedrig. Man sollte sich allerdings vor einer Anhebung der Steuersätze oder vor der Einführung neuer Steuern hüten, da diese Massnahmen zweifellos nur sehr kurzfristig Gewinne einbrächten und sich darüber hinaus als kontraproduktiv erweisen würden. Die Verwaltungsreformen müssen, namentlich mit dem Ausbau der Steuerbehörde ATM, fortgesetzt werden. Ferner müssen Schwächen bei der Umsetzung des bestehenden Systems, die den Produktionssektor benachteiligen (z.B. bei der Rückzahlung der Mehrwertsteuerkredite), behoben werden. Sodann ist auch ein Wiederausgleich des
Systems durch die Neuorganisation des Steuerbefreiungssystems notwendig. Die recht aggressive Politik der Steuernachlässe, um das Programm zur Förderung ausländischer Investitionen zu lancieren, sollte regelmässig evaluiert werden. Schliesslich werden die Steuerreformen durch den Dezentralisierungsprozess, der sich gleichzeitig abspielt und der eine Neufestlegung der Steuerhoheit zwischen Verwaltungsebenen nach sich zieht, komplexer gestaltet. An diesen vielfältigen Fronten sind das SECO und die anderen in dem Bereich tätigen Partner bereit, den Behörden Mosambiks weiterhin ihre Unterstützung zukommen zu lassen. Mit einem Zeithorizont von fünf bis zehn Jahren rechnet man demnach mit einer Erhöhung des Steuerdrucks bis auf 15 Prozent, sodann auf 20 Prozent des BIP. 